\title{
Measuring Success in Psychotherapy Trials: The Challenge of Choosing the Adequate Control Condition
}

\author{
Stephan Zipfel Florian Junne Katrin E. Giel
}

Department of Psychosomatic Medicine and Psychotherapy, University of Tuebingen, Tuebingen, Germany

\section{The Art of Finding the Right Control Condition for Psychotherapy Studies}

Various forms of psychotherapies have been shown to be efficacious and cost-effective for a wide spectrum of mental disorders. The reference policy of the American Psychological Association (APA) developed for Evidence-Based Psychotherapies (EBP) [1] follows the guidelines by Sackett et al. [2]. This APA policy emphasizes the need to integrate the best available research with clinical expertise in the context of the patient's culture, individual characteristics, and personal preferences [3]. Concerns have been raised about the generalizability of the findings from randomized controlled psychotherapy trials, since the conditions and characteristics of such efficacy trials differ significantly from routine clinical practice (effectiveness perspective). This is all the more important in the field of psychotherapy research, as there are factors to patient success, such as the individual patientpsychotherapist relationship, which can influence the observed outcomes without directly depending on the novel technique or therapeutic approach under investigation. In contrast, double-blind randomized controlled trials (RCTs) are still considered as the gold standard for medical research in general. However, behavioral interventions, and in particular disorder-specific psychotherapy

karger@karger.com

(c) 2020 S. Karger AG, Basel

www.karger.com/pps

Karger! techniques, differ in their complex nature in many ways from the classic application of double-blind RCTs seen in drug trials.

Recently, we have discussed the issue of possible placebo effects in psychotherapy [4] with respect to: (a) nonspecific factors shared with drug therapy (context factors), (b) nonspecific factors shared among all psychotherapy traditions (common factors), (c) specific placebo-controlled options with different psychotherapy modalities, and finally (d) nonspecific control options for the specific placebo effect in psychotherapy. Additionally, there are also particular differences, for example, doubleblinding cannot be applied and the control condition is usually not just an inert placebo pill.

Gold et al. [5] have developed a decision framework for control conditions for RCTs of behavioral interventions in psychiatry. They suggested a list of frequently used control conditions in trials of behavioral interventions (see Table 1).

As a clear implication, the authors explain that investigators should: (a) describe in detail and (b) justify their choice of the control conditions for a planned trial explicitly in their trial protocol and subsequently in the trial publication. When choosing a control group population, the respective risks should be considered for each trial phase. They stratified their decision framework based on

Prof. Dr. Stephan Zipfel

Department of Psychosomatic Medicine and Psychotherapy

Medical University Hospital Tuebingen

Osianderstrasse 5, DE-72076 Tuebingen (Germany)

stephan.zipfel@med.uni-tuebingen.de 
Table 1. Frequently used control conditions in behavioral intervention trials (adapted from Gold et al. [5])

\begin{tabular}{|c|c|}
\hline Control condition & Short description \\
\hline Active comparator & $\begin{array}{l}\text { A treatment that has an evidence base to support its efficacy but is different from the experimental } \\
\text { treatment }\end{array}$ \\
\hline Minimal treatment control & Treatments that entail fewer than four sessions \\
\hline $\begin{array}{l}\text { Nonspecific factors } \\
\text { component control }\end{array}$ & $\begin{array}{l}\text { Includes time with a therapist of the same duration and frequency as the experimental treatment, but } \\
\text { no exercises or techniques regarded as therapeutic. This control condition often includes educational } \\
\text { sessions during which patients are informed only about treatments available or self-aid options }\end{array}$ \\
\hline No-treatment control & Contains no study treatment and is not done in a setting in which treatment would be available \\
\hline Patient's choice & $\begin{array}{l}\text { Patients can choose freely between treatments offered in a trial (e.g., one of several types of } \\
\text { psychotherapy or between psychotherapy and medication) }\end{array}$ \\
\hline Pill placebo & $\begin{array}{l}\text { A placebo pill is given to the control group, which does not receive the experimental behavioral } \\
\text { treatment }\end{array}$ \\
\hline Waitlist control & $\begin{array}{l}\text { No treatment is provided during the experimental treatment period, but the experimental treatment is } \\
\text { offered after posttreatment assessment }\end{array}$ \\
\hline
\end{tabular}

low, intermediate, and high participation risk. The authors also claim that no behavioral treatment should be included in treatment guidelines if its effectiveness is supported only by trials consisting of a waitlist control group or by meta-analytic evidence conducted on such trials. Finally, the authors stress the fact that indirect comparisons of efficacy and effectiveness based on trials of behavioral and pharmacological therapies, tested against different control conditions, are problematic.

As a conclusion, the authors of this framework demand that for the choice of a control condition in a behavioral intervention, the type of intervention, patient population, available resources, and finally the respective trial phase should be clearly considered and reported.

In their methodological recommendation for trials of psychological interventions, Guidi et al. [6] reflect the crucial role of the study design and the choice of the respective control group condition. In line with Gold et al. [5], they state that a waiting list control condition may be only suitable for preliminary testing of an experimental procedure and for pilot studies. In addition, the authors point out that treatment as usual (TAU) control conditions are often not properly manualized, as well as insufficiently monitored and supervised. A potential solution for this aspect might be the optimized TAU condition
(TAU-O) where researchers try to include and monitor the best available evidence derived by the actual treatment guidelines for specific disorders [7, 8]. However, for patients with newly developed psychotherapy conditions undertaking such a structured TAU-O control condition could be a particularly strong competitor and may lead to two active psychological treatments with nonsignificant differences in statistical tests. On the other hand, such a study design makes participation in the study ultimately more attractive for participating patients and may help to minimize drop-out rates in the control arm.

Both, Gold et al. [5] and Guidi et al. [6] also line out the possibility to use a nonspecific component control group. Such a control condition includes the same dosage of therapy sessions as in the experimental treatment, but no ingredients regarded as therapeutic (see Table 1). This has been sometimes also referred to as an "attention placebo," while it should be considered that such conditions might suffer from lower acceptance and high dropout rates [6]. Moreover, Guidi et al. [6] also emphasize that it is important to differentiate so-called clinical management control conditions from nonspecific factor component control (see below for an in-depth example and discussion of such a clinical management control condition). 
Based on the recommendation from Guidi et al. [6] dismantling studies (i.e., comparing a full intervention to a control intervention where specific components of the full intervention are not applied) is another important option when designing psychotherapy trials. A psychotherapeutic approach is likely to include multiple treatment components. As a result, dismantling studies may determine which components may yield additional value to the treatment and again the crucial question is, which component condition serves as control group.

A specific approach has to be considered when studying interactions of experimental psychotherapy with pharmacology. There are four models of interventions involved: (1) addition (the effect of two interactions combined equals the sum of their individual effects), (2) potentiation (the effect of two interventions combined is greater than the sum of their individual effects), (3) inhibition (the effect of two interventions combined is less than each individual effect), and (4) reciprocation (the effect of two interventions combined equals the individual effect of the more potent intervention) (see [6]).

In the future, studies with adaptive designs and interventions may play a more prominent role in the times of individualized and precision therapy. Such a targeted and gradual intervention could then focus even more precisely on specific characteristics of the individual patient, which could come from the entire spectrum of bio-psycho-social factors and also include former patients' response to first-line treatment. Such complex and individualized study approaches could also benefit from the rapidly growing research on artificial intelligence in the near future. However, this will require even broader interdisciplinary expertise, which will also demand the involvement of data scientists [9].

In addition, the distinct differences in healthcare systems between different countries should also be taken into account in the consideration of an adequate control condition in the future. Especially with regard to the question of financing psychotherapy, this can depend on the socioeconomic level of the country, but also on very different traditions. In Germany, for example, there is a long tradition of psychosomatic medicine with a broad range of inpatient and day care services [10]. In addition, Germany has a long tradition of financing outpatient psychotherapy, including psychoanalytic, psychodynamic, as well as cognitive behavior psychotherapy. In such a treatment environment, TAU can be much more intensive than in other healthcare systems, as these services may not be available as TAU control conditions.

Choosing the Adequate Control Condition in Psychotherapy Trials
Another factor that is usually not taken into account is the interaction of diagnostics and therapy. Particularly in the case of those mental disorders for which a structured monitoring of symptoms is an integral part of therapy, an intensive process and multistep outcome monitoring can also have a certain therapeutic effect. Such aspects of selfmonitoring should be discussed at least in the case of significant control group effects [11].

\section{Challenges for Trials Investigating Digitally Enhanced Psychotherapy}

In recent years, there has been an increasing challenge for providing digital health [12] and subsequently also in the field of mental healthcare [13]. As a consequence, the challenge for the design of control conditions of psychotherapeutic interventions can be seen in the complex mix of components in the context of digitally enhanced treatment approaches. For example, if active treatment conditions are delivered as a combination of face-to-face psychotherapy with an (intersession) e-health component, such as an online application of mindfulness-based stress reduction (MBSR), it is of crucial importance to monitor control group participants for any use of such (potentially confounding) online applications that are available on the market. However, monitoring such use of confounding applications may be of limited value because it is usually reliant on self-reporting. Study participants may, for example, not recognize any use with potentially confounding applications. Although technically feasible, the alternative of web tracking the use of potentially confounding (online) applications by control participants faces serious ethical and data safety constraints.

\section{When the Control Condition Is the More Effective Intervention}

In this context, it becomes particularly interesting when the supposed control condition is significantly more successful than the actual experimental psychotherapeutic intervention.

One specific explanation for such a constellation might be that the experimental treatment entails adverse effects [14]. However, this can also happen under active control conditions, which are particularly close to the mechanisms of action of the disorder and sometimes include additional optimization of the therapy according to current guidelines. 
In the field of eating disorders, this has been seen, for example, with the so-called Specialist Supportive Clinical Management (SSCM). SSCM was initially developed as an atheoretical, unspecific active control condition for an RCT on the efficacy of cognitive behavior therapy versus interpersonal psychotherapy in the treatment of anorexia nervosa (AN) [15]. In this trial, it proved more efficacious than the two specific interventions and has since been used in other RCTs on AN treatment and has even been included in national treatment guidelines [16]. It has been speculated that SSCM might work as it incorporates a few "general" components which induce change, i.e., a focus on core pathologies while using a supportive approach and a positive working alliance. Especially in the case of severe disorders like $\mathrm{AN}$, where prognosis is still limited and novel treatment approaches are urgently needed [7], it is actually highly beneficial if treatment options can be broadened by an intervention which unexpectedly targets the disorder.

This particular constellation brings us to the current study by van Passel et al. [17] in this issue of Psychotherapy and Psychosomatics. They present data from an RCT in which they have investigated the efficacy of cognitive remediation therapy (CRT) as an adjuvant therapy prior to TAU for obsessive compulsive disorder (OCD) and AN. The rationale behind this trial entails that patients suffering from both disorders typically show cognitive inflexibility which might reduce the potential benefit from TAU, which targets the core symptoms of the disorder. In a rigorous RCT study design, the group has conducted CRT in a decent sample size of OCD and AN patients, while a central innovative design aspect lies in the implementation of a novel active control condition called SAT which closely resembles CRT without addressing aspects of cognitive inflexibility. Other strengths of the trial include comparably low immediate dropout rates from the adjuvant treatments, suggesting acceptability of the interventions, as well as a 12-month-follow-up assessment. Taking a look at the primary outcome, in the long run, most of the patients who were available for follow-up assessments were improved and many remained unchanged or stable. However, there was no evidence for CRT to enhance the efficacy of TAU. On the contrary, there was even a trend for the active control condition SAT to result in superior outcomes in combination with TAU for OCD patients. From this perspective, the trial by van Passel et al. [17] is at first glance a so-called negative study, as the trialed intervention was not beneficial in terms of the primary outcome. Nevertheless, the trial is promising as the ac- tive control condition seems to have more potential than initially expected and should be investigated further, similarly to SSCM.

\section{Are Negative Studies Really Negative?}

There is yet another aspect which might have contributed to the control condition being superior in the Van Passel trial (and other trials before), which has also been emphasized recently in The Lancet Psychiatry [18]. It is the choice and especially the assessment of the primary outcome that can be very challenging in the field of mental health where there is often no clear biomarker or "objective" measure available and that disorders might be characterized by different key symptoms [18]. This may lead to circumstances in which a trial turns out "negative" on an outcome which has been chosen more or less arbitrarily from different potential equivalent outcomes. Especially in the field of investigating novel interventions for mental diseases, this might imply that secondary outcomes should gain much more attention. They might also tell a lot on whether an intervention might be beneficial for patients. This has also led to the call for integrating the perspectives of individuals with lived experience into the development of clinical trials [18] as they might help to choose outcomes beyond the mere "RCT logic" which make a difference in the lives of individuals living with mental health issues and their carers.

\section{Conclusion}

Together with the Lancet Psychiatry Commission on psychological treatments research in Tomorrow's Science [19], we are convinced that dedicated effort is needed for conducting state-of-the-art trials to assess psychological therapies and inform policy and practice. Accordingly, the design and conduct of these trials warrants scrutiny and quality improvement. An important factor is the selection of an adequate and suitable control condition. This is especially the case for the complex interplay of active and confounding components within digitally enhanced psychotherapeutic interventions. Clinical researchers should be trained even better and earlier in this growing aspect of clinical research. Meanwhile, models for such systematic training have been tested and proven [20]. 


\section{References}

1 APA Presidential Task Force on EvidenceBased Practice in Psychology. Evidence-based practise in psychology. Am Psychol. 2006 May-Jun;61(4):271-85.

2 Sackett DL, Rosenberg WM, Gray JA, Haynes RB, Richardson WS. Evidence based medicine: what it is and what it isn't. BMJ. 1996 Jan; 312(7023):71-2.

3 Cook SC, Schwartz AC, Kaslow NJ. EvidenceBased Psychotherapy: advantages and Challenges. Neurotherapeutics. 2017 Jul;14(3): 537-45.

4 Enck P, Zipfel S. Placebo Effects in Psychotherapy: A Framework. Front Psychiatry. 2019 Jun; 10:456.

5 Gold SM, Enck P, Hasselmann H, Friede T, Hegerl U, Mohr DC, et al. Control conditions for randomised trials of behavioural interventions in psychiatry: a decision framework. Lancet Psychiatry. 2017 Sep; 4(9):725-32.

6 Guidi J, Brakemeier EL, Bockting CL, Cosci F, Cuijpers P, Jarrett RB, et al. Methodological Recommendations for Trials of Psychological Interventions. Psychother Psychosom. 2018; 87(5):276-84

7 Zipfel S, Giel KE, Bulik CM, Hay P, Schmidt U. Anorexia nervosa: aetiology, assessment, and treatment. Lancet Psychiatry. 2015 Dec; 2(12):1099-111.

8 Zipfel S, Wild B, Groß G, Friederich HC, Teufel M, Schellberg D, et al.; ANTOP study group. Focal psychodynamic therapy, cognitive behaviour therapy, and optimised treatment as usual in outpatients with anorexia nervosa (ANTOP study): randomised controlled trial. Lancet. 2014 Jan;383(9912):12737.

9 Miner AS, Shah N, Bullock KD, Arnow BA, Bailenson J, Hancock J. Key Considerations for Incorporating Conversational AI in Psychotherapy. Front Psychiatry. 2019 Oct;10: 746.

10 Zipfel S, Herzog W, Kruse J, Henningsen P. Psychosomatic Medicine in Germany: More Timely than Ever. Psychother Psychosom. 2016;85(5):262-9.

11 Schag K, Rennhak SK, Leehr EJ, Skoda EM, Becker S, Bethge W, et al. IMPULS: Impulsivity-Focused Group Intervention to Reduce Binge Eating Episodes in Patients with Binge Eating Disorder - A Randomised Controlled Trial. Psychother Psychosom. 2019;88(3): 141-53.

12 Duggal R, Brindle I, Bagenal J. Digital healthcare: regulating the revolution. BMJ. 2018 Jan; 360:k6.

13 Bucci S, Schwannauer M, Berry N. The digital revolution and its impact on mental health care. Psychol Psychother. 2019 Jun;92(2): 277-97.

14 Fava GA, Benasi G, Cosci F. The potential role of iatrogenic comorbidity in the interaction between pharmacotherapy and psychothera- py in anxiety disorders. Verhaltenstherapie. 2017;27(4):265-70.

15 McIntosh VV, Jordan J, Carter FA, Luty SE, McKenzie JM, Bulik CM, et al. Three psychotherapies for anorexia nervosa: a randomized, controlled trial. Am J Psychiatry. 2005 Apr; 162(4):741-7.

16 Jordan J, McIntosh VV, Bulik CM. Specialist Supportive Clinical Management for anorexia nervosa: what it is (and what it is not). Australas Psychiatry. 2019 Sep; 1039856219875024

17 van Passel B, Danner UN, Dingemans AE, Aarts E, Sternheim LC, Becker ES, et al. Cognitive Remediation Therapy Does Not Enhance Treatment Effect in Obsessive-Compulsive Disorder and Anorexia Nervosa: A Randomized Controlled Trial. Psychother Psychosom. 2020 Feb;1-14. doi: 10.1159/ 000505733.

18 The Lancet Psychiatry. Measuring success: the problem with primary outcomes. Lancet Psychiatry. 2020 Jan;7(1):1.

19 Holmes EA, Ghaderi A, Harmer CJ, Ramchandani PG, Cuijpers P, Morrison AP, et al. The Lancet Psychiatry Commission on psychological treatments research in tomorrow's science. Lancet Psychiatry. 2018 Mar;5(3): 237-86.

20 Monzer N, Herzog W, Löwe B, Zipfel S, Henningsen $\mathrm{P}$, Rose $\mathrm{M}$, et al. Reviving the Clinician Scientist: A Best Practice Model. Psychother Psychosom. 2019;88(2):114-5. 